\title{
Viewpoint
}

\section{Extragalactic diffuse gamma-ray emission at high energies}

\author{
Uwe Oberlack \\ Department of Physics and Astronomy, Rice University, Houston TX, USA
}

Published March 8, 2010

New measurements with the Fermi Large Area Telescope extend our knowledge of the extragalactic diffuse gamma-ray background and may help resolve the question of its origins.

Subject Areas: Particles and Fields, Astrophysics

\author{
A Viewpoint on: \\ Spectrum of the Isotropic Diffuse Gamma-Ray Emission Derived from First-Year Fermi Large Area Telescope \\ Data \\ A. A. Abdo et al. (Fermi LAT Collaboration) \\ Phys. Rev. Lett. 104, 101101 (2010) - Published March 8, 2010
}

A diffuse and approximately isotropic high-energy photon background has been known to exist in the $\mathrm{x}$ ray regime since 1962 [1] and a similar background was observed by the SAS-2 satellite at high energies [2]. Generally, isotropic or nearly isotropic emission may arise from extragalactic sources or an extended halo around the galaxy. It may result from superposition of many unresolved point sources or truly diffuse emission. The extragalactic component is usually referred to as the cosmic x-ray background and the cosmic gamma-ray background, depending on energy. The Fermi LAT Collaboration has now published in Physical Review Letters[3] their first results on the isotropic diffuse gamma-ray emission measured with the Fermi Large Area Telescope (LAT) in the energy range from $200 \mathrm{MeV}$ to $100 \mathrm{GeV}$. The new result, based on the initial ten months of science observations, significantly improves and extends our knowledge of the isotropic diffuse emission at the high-energy end. The observed emission is softer and lower in intensity than measurements with EGRET had indicated previously [4]. Fermi's observed spectrum is consistent with a single power-law description over nearly three orders of magnitude in energy, and hence removes an apparent change in spectral shape around $\sim 2-3 \mathrm{GeV}$, observed with EGRET. This is significant in discussing possible sources.

The measurement of the isotropic emission has been challenging due to two competing contributions: instrumental background and diffuse emission from galactic or local foregrounds. In the hard x-ray $(>10 \mathrm{keV})$ to high-energy gamma-ray regime, now extended up to $100 \mathrm{GeV}$, the lack of focusing apertures leads to substantial instrumental backgrounds due to cosmic-ray interactions either directly or indirectly through secondary processes. Astrophysical foregrounds vary with energy-with bremsstrahlung, inverse Compton scattering of cosmic ray electrons on the interstellar radiation

DOI: $10.1103 /$ Physics. 3.21

URL: http://link.aps.org/doi/10.1103/Physics.3.21 field, and cosmic-ray interactions with interstellar gas dominating at increasingly high energies. Suborbital measurements and measurements using earth occultation techniques are also hampered by the glow of earth's atmosphere due to cosmic-ray interactions.

The Fermi LAT data analysis had to fight the same nuisances: instrumental background and astrophysical foreground. The instrumental background was reduced to acceptable levels using stringent data cuts, with some compromising on instrumental sensitivity. The astrophysical foreground, however, could only be reduced by choosing galactic latitudes greater than $10^{\circ}$ and accounting for known point sources. The remaining diffuse foreground had to be modeled carefully, and leaves the greatest systematic uncertainty. The foreground diffuse galactic emission is modeled by a cosmic-ray propagation model, with parameters optimized to fit various cosmic-ray and gamma-ray data sets, using maps of hydrogen in its various phases in the interstellar medium to fit gamma-ray emission from cosmic-ray interactions. One potential problem includes the lack of a systematic survey of molecular hydrogen at high galactic latitudes, even though no major cloud formations are known to exist outside the covered survey area. The good linear correlation of high-energy gamma-ray emission with the known column densities of atomic hydrogen observed by the Fermi team [5] support the idea that atomic hydrogen may be sufficient to consider. This analysis can also be used to estimate the cosmic diffuse background in a less model-dependent way (even though the same atomic hydrogen maps enter both analyses). An expected overestimate of the extragalactic component from this analysis is observed. The modeling of the diffuse galactic component, with numerous free parameters, including the halo size for cosmic-ray electrons, is a concern. The fits in subregions of the sky, provided as an electronic supplement, show the fit al- 


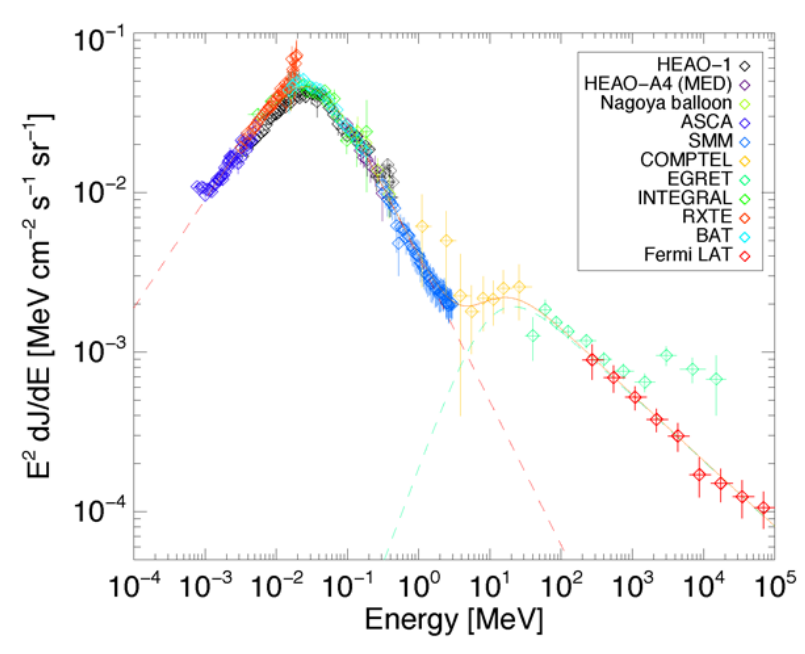

FIG. 1: The extragalactic diffuse high-energy background shown as a plot of $E^{2}$ times photon number spectrum versus energy using the compilation of Ajello et al.[6]. Two components are apparent, each described by a broken power law, showing two maxima in emitted energy around $25 \mathrm{keV}$ and $20 \mathrm{MeV}$. The solid line indicates the sum of both fitted components. The new Fermi LAT data points are at the highest energies, indicated in red.

ways passing through the error bars of all data points, possibly indicating an overfit of the data. Interestingly, Fermi LAT also observed some foreground emission from the Solar System, which will be explored further.

What are the sources of this diffuse high-energy emission? Figure 1 shows the reproduction of a compilation [6] of data from the x-ray to gamma-ray regime, with the new Fermi LAT data added for illustration. The vertical logarithmic axis is the photon intensity scaled with energy squared $\left(E^{2}\right)$, and in such a plot, the photon number spectrum with power-law index -2 would be represented by a horizontal line. This representation indicates the power emitted in logarithmic intervals. (See Ref. [7] for a critical discussion of these units.) It is remarkable that the spectrum can be reasonably described (within the uncertainties of combining more than ten different instruments) by just two distinct parts across eight orders of magnitude in energy. The lower energy component, a continuous extension of the cosmic x-ray background, dominates below $\sim 1 \mathrm{MeV}$ and can be described by a broken power law

$$
\frac{d J}{d E}=\frac{C}{\left[\left(E / E_{b}\right)^{\Gamma_{1}}+\left(E / E_{b}\right)^{\Gamma_{2}}\right]},
$$

with indices $\Gamma_{1}=1.32 \pm 0.018$ and $\Gamma_{2}=$ $2.88 \pm 0.015$ [6], peaking around $\sim 25 \mathrm{keV}$. The photon number spectrum $d J / d E$ is in units of photons $\mathrm{cm}^{-2} \mathrm{~s}^{-1} \mathrm{sr}^{-1} \mathrm{MeV}^{-1}$. (Including data from the Swift-XRT, Moretti et al.[8] find $\Gamma_{1}=1.41 \pm 0.02$.) The higher energy component, peaking around $\sim 20 \mathrm{MeV}$, can also be described by a broken power law, with one power law index around $\Gamma_{3} \approx 1$, poorly constrained by the data between $\sim 1 \mathrm{MeV}$ and $\sim 20 \mathrm{MeV}$, and the second index of $\Gamma_{4}=2.41 \pm 0.05$ determined by the Fermi data. An approximate composite fit as shown in Fig. 1 indicates that the power law index $\Gamma_{2}$ may be somewhat larger (steeper) than when fitted individually.

The cosmic x-ray background is generally understood as the cumulative emission from unobscured active galactic nuclei (AGN) in the soft $x$-ray regime, where below $2 \mathrm{keV}$ the emission can be nearly fully resolved into individual sources, with increasing contribution from obscured, Compton-thick AGN in the hard x-ray regime [9], even though star-forming galaxies may also add to the observed emission [8]. For the cosmic gamma-ray background, the identification with sources is not settled yet. Estimates on the contribution of, again, AGN vary from the $\sim 10 \%$ level [10] for AGN of the "blazar" type (with jets pointing towards us under small angles) to being the main source, based on nonblazar types dominating up to $\sim 100 \mathrm{MeV}$ and blazar types dominating in the Fermi energy range [11]. On the other hand, the observed galactic foreground emission suggests that cosmic rays in star-forming galaxies may be substantial contributors as well [10]. Truly diffuse emission from dark matter annihilation is usually expected to be small, but could be boosted in certain scenarios [12]. The new Fermi measurement, being compatible with a single power law, adds support to the case of a single dominant source class.

While this result is a big step forward, we are only beginning to explore the ever richer Fermi database to understand the cosmic gamma-ray background. With increasing exposure, an increasing number of point sources will be detected and studied, and correlations of the extragalactic diffuse emission in smaller subfields with known populations will provide additional input. Limitations are likely to arise from the relatively coarse angular resolution of Fermi LAT, which features a point spread function of close to $3^{\circ}$ diameter in the $95 \%$ envelope at $1 \mathrm{GeV}$, and $\sim 0.6^{\circ}$ at $10 \mathrm{GeV}$ [13], and the uncertainty in modeling the galactic halo diffuse foreground, resulting from inverse Compton scattering.

\section{Acknowledgment}

I would like to thank Marco Ajello for kindly providing his data compilation for the figure.

\section{References}

[1] R. Giacconi, H. Gursky, F. R. Paolini, and B. B. Rossi, Phys. Rev. Lett. 9, 439 (1962).

[2] D. J. Thompson and C. E. Fichtel, Astron. Astrophys. 109, 352 (1982).

[3] A. A. Abdo et al. (Fermi LAT Collaboration), Phys. Rev. Lett. 104, 101101 (2010).

(C) 2010 American Physical Society 
[4] P. Sreekumar et al., Astrophys. J. 494, 523 (1998); A. W. Strong, I. V. Moskalenko, and O. Reimer, Astrophys. J. 613, 956 (2004).

[5] A. Abdo et al., Astrophys. J. 703, 1249 (2009).

[6] M. Ajello et al., Astrophys. J. 689, 666 (2008).

[7] R. C. Henry, Astrophys. J. 516, L49 (1999).

[8] A. Moretti et al., Astron. Astrophys. 493, 501 (2009).

[9] R. Gilli, A. Comastri, and G. Hasinger, Astron. Astrophys. 463, 79 (2007).
[10] C. D. Dermer, AIP Conf. Proc. 921, 122 (2007) .

[11] Y. Inoue and T. Totani, Astrophys. J. 702, 523 (2009).

[12] M. Taoso, S. Ando, G. Bertone, and S. Profumo, Phys. Rev. D 79, 043521 (2009).

[13] R. Rando(for Fermi LAT Collaboration), contribution to the 31st International Cosmic Ray Conference, Lodz, Poland, July 2009; arXiv:0907.0626v1 .

\section{About the Author}

\section{Uwe Oberlack}

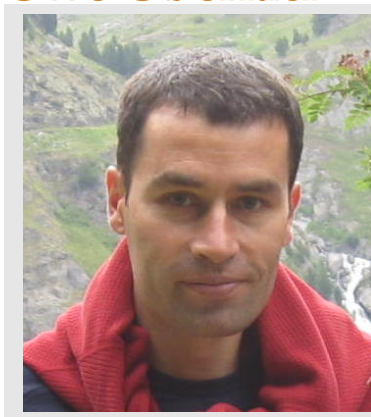

Uwe Oberlack studied physics at the Technical University in Munich, Germany, from which he also received his Ph.D. for research performed at the Max Planck Institute of Extraterrestrial Physics with the COMPTEL gamma-ray telescope aboard the Compton Gamma-Ray Observatory. For his thesis research on radioactive ${ }^{26} \mathrm{Al}$, including the generation of the first all-sky maps in this line, he was awarded the Otto Hahn Medal by the Max Planck Society in 1998. After post-doctoral research at Columbia University, working on a novel balloon-borne Compton telescope, he moved to Rice University in Houston, TX, where he has been on the faculty since 2001. While his research focus has since shifted to the direct search for dark matter with the XENON project, he continues to pursue interests in gamma-ray astronomy and its instrumentation. 